\title{
Bioelectrical impedance phase angle as a prognostic indicator in advanced pancreatic cancer
}

\author{
Digant Gupta*, Christopher G. Lis, Sadie L. Dahlk, Pankaj G. Vashi, James F. Grutsch and \\ Carolyn A. Lammersfeld \\ Cancer Treatment Centers of America ${ }^{\circledR}$ (CTCA) at Midwestern Regional Medical Center, 2520 Elisha Avenue, Zion, \\ IL 60099, USA
}

(Received 11 May 2004 - Revised 17 August 2004 - Accepted 17 August 2004)

\begin{abstract}
Bioelectrical impedance analysis (BIA) is an easy-to-use, non-invasive and reproducible technique to evaluate changes in body composition and nutritional status. Phase angle, determined by BIA, has been found to be a prognostic indicator in several chronic conditions, such as HIV, liver cirrhosis, chronic obstructive pulmonary disease and lung cancer, and in patients undergoing dialysis. The present study investigated the prognostic role of phase angle in advanced pancreatic cancer. We evaluated a case series of fifty-eight stage IV pancreatic cancer patients treated at Cancer Treatment Centers of America ${ }^{\circledR}$ at Midwestern Regional Medical Center (Zion, IL, USA) between January 2000 and July 2003. BIA was conducted on all patients using a bioelectrical impedance analyser that operated at $50 \mathrm{kHz}$. The phase angle was calculated as capacitance $(\mathrm{Xc}) /$ resistance $(\mathrm{R})$ and expressed in degrees. The Kaplan-Meier method was used to calculate survival. Cox proportional hazard models were constructed to evaluate the prognostic effect of phase angle independent of other clinical and nutritional variables. The correlations between phase angle and traditional nutritional measures were evaluated using Pearson and Spearman coefficients. Patients with phase angle $<5.0^{\circ}$ had a median survival time of 6.3 (95\% CI 3.5, 9.2) months $(n$ 29), while those with phase angle $>5.0^{\circ}$ had a median survival time of 10.2 (95\% CI 9.6, 10.8) months $(n$ 29); this difference was statistically significant $(P=0.02)$. The present study demonstrates that phase angle is a strong prognostic indicator in advanced pancreatic cancer. Similar studies in other cancer settings with larger sample sizes are needed to further validate the prognostic significance of the phase angle.
\end{abstract}

Pancreatic cancer: Bioelectrical impedance analysis: Phase angle: Survival time

Pancreatic cancer is the fourth leading cause of cancer deaths in the USA (Rocha Lima \& Centeno, 2002). Despite considerable progress in the areas of epidemiology, molecular genetics, diagnostics, operative techniques and overall patient management, the overall 5-year survival rate for pancreatic cancer is $4.4 \%$, whereas distant stage pancreatic cancer is merely $1.6 \%$ (Rocha Lima \& Centeno, 2002; Bohmig \& Rosewicz, 2004).

Progressive weight loss and nutritional deterioration are commonly found in patients with pancreatic cancer (Ottery, 1996) and a majority of patients are already in a state of malnutrition on admission (Higashiguchi et al. 1988). According to the National Cancer Institute's Nutrition in Cancer Care guidelines, timely identification and treatment of nutrition problems may improve a cancer patient's prognosis by helping the patient to gain or maintain weight, improving the patient's response to therapy and reducing the complications of treatment. As a result, identifying and treating malnutrition early in the course of the pancreatic cancer is critical to achieving favourable patient outcomes. However, this has been a significant challenge for oncologists because of the lack of a valid and reliable tool to measure nutritional status.

Historically, nutritional status has been evaluated by various objective measures, including anthropometric (e.g. weight change, mid-arm muscle circumference, triceps skinfold thickness) and laboratory (serum albumin, transferrin assays and $\mathrm{N}$ balance studies) measurements. In the clinical setting, anthropometric methods are not ideal, because they are time-consuming and difficult to perform, especially in bed-ridden patients. Some of the objective measures such as serum albumin are likely to be influenced by many nonnutritional factors (Bauer et al. 2002; Carney \& Meguid, 2002; Waitzberg \& Correia, 2003). The interpretation of these objective measures is often difficult because non-nutritional factors, such as hydration state and disease process, can obscure the effects of actual nutrient deprivation (Detsky et al. 1984). Furthermore, some objective indicators such as serum albumin have long half-lives, making it difficult to assess changes in the nutritional status over a short period of time. A less-used tool to assess nutritional status - bioelectrical impedance analysis (BIA) - exists, and may overcome some of these challenges. 
BIA is an easy-to-use, non-invasive and reproducible technique to evaluate changes in body composition. BIA has been validated for the assessment of body composition and nutritional status in a variety of patient populations including cancer patients (Lukaski et al. 1985; Fredrix et al. 1990; Adami et al. 1993; Azcue et al. 1993; Marken Lichtenbelt et al. 1994; Chertow et al. 1995; Simons et al. 1995; Pencharz \& Azcue, 1996; Chertow et al. 1997; Pupim et al. 1999; Nagano et al. 2000; Fein et al. 2002; Desport et al. 2003; Sarhill et al. 2003). BIA is based on the principle that a fixed, low-voltage, high-frequency alternating current introduced into the human body is conducted almost completely through the fluid compartment of the fat-free mass (Simons et al. 1995). BIA measures body component resistance (R) and capacitance $(\mathrm{Xc})$ by recording a voltage drop in applied current (Zarowitz \& Pilla, 1989). Bioelectric resistance is the pure opposition of a biological conductor to the flow of an alternating electric current, whereas capacitance is the resistive effect due to capacitance produced by tissue interfaces and cell membranes. Capacitance causes the current to lag behind the voltage, creating a phase shift. This shift is quantified geometrically as the angular transformation of the capacitance:resistance ratio, or phase angle (Baumgartner et al. 1988).

The phase angle reflects the relative contributions of fluid (resistance) and cellular membranes (capacitance) of the human body. By definition, the phase angle is positively associated with capacitance and negatively associated with resistance (Baumgartner et al. 1988). The phase angle can also be interpreted as an indicator of water distribution between the extra- and intra-cellular spaces, one of the most sensitive indicators of malnutrition (Talluri et al. 1999; Schwenk et al. 2000). The phase angle has been found to be a prognostic marker in several clinical conditions where cell membrane integrity is compromised and alterations in fluid balance are noted, such as HIV infection, liver cirrhosis, chronic obstructive pulmonary disease, haemodialysis and sepsis (Ott et al. 1995; Maggiore et al. 1996; Schwenk et al. 1998; Faisy et al. 2000; Schwenk et al. 2000; Selberg \& Selberg, 2002). In the current study, we investigated whether the phase angle could provide any prognostic information in patients with advanced pancreatic cancer. The primary objective of the present study was to evaluate the association of the BIA-derived phase angle with overall survival in patients with stage IV pancreatic cancer. The secondary objective was to investigate the relationship between the phase angle and traditional indicators of nutritional status.

\section{Materials and methods}

\section{Subjects}

A retrospective chart review was performed on a consecutive case series of fifty-eight stage IV pancreatic cancer patients treated at Cancer Treatment Centers of America ${ }^{\circledR}$ at the Midwestern Regional Medical Center (Zion, IL, USA) between January 2000 and July 2003. The patients were identified from the Midwestern Regional Medical Center tumour registry. All tumours were adenocarcinomas.

\section{Nutritional assessment}

All patients underwent a baseline nutritional assessment by a registered dietitian, including laboratory measurements of serum albumin, prealbumin and transferrin, subjective global assessment and BIA. BIA was performed using a bioelectrical impedance analyser (model BIA101Q; RJL Systems Inc., Clinton Township, MI, USA). BIA was conducted while patients were lying supine on a bed or examination table, with legs apart and arms not touching the torso. All evaluations were conducted on the patients' right side using the four surface standard electrode (tetrapolar) technique on the hand and foot (Lukaski et al. 1985). One electrode was placed on an imaginary line from the protruding bone on the little finger side of the wrist to bisect the ulnar head on the right hand. Another electrode was placed below the knuckle of the middle finger on the right hand. A third electrode was placed on an imaginary line from the protruding bone on the big toe side on the ankle, to bisect the medial malleolus of the right foot. The fourth and final electrode was placed just below the middle toe of the right foot. Resistance $(\mathrm{R})$ and capacitance $(\mathrm{Xc})$ were directly measured in ohms $(\Omega)$ at $50 \mathrm{kHz}, 800 \mu \mathrm{A}$ using the BIA (RJL Systems Inc.). One assessment of resistance $(\mathrm{R})$ and capacitance $(\mathrm{Xc})$ was made. The phase angle was calculated using the following equation:

$$
\text { phase angle }=(\text { resistance } / \text { capacitance }) \times(180 / \pi) .
$$

\section{Statistical analysis}

All data were analysed using SPSS 11.5 (SPSS Inc., Chicago, IL, USA). Overall patient survival was defined as the time interval between the date of first patient visit to the hospital and the date of death from any cause or the date of last contact or last known to be alive. The Kaplan-Meier or product-limit method was used to calculate survival time. The log rank test statistic was used to evaluate the equality of survival distributions across different strata. A difference was considered to be statistically significant if $P \leq 0 \cdot 05$. Survival time was also evaluated using univariate and multivariate Cox regression analysis. Variables evaluated included the phase angle, age at diagnosis, previous treatment history, serum albumin, serum prealbumin and serum transferrin. Variables in the multivariate models were selected with the backward elimination likelihood ratio method and with thresholds of $\alpha<0.05$ for entry and $\alpha \geq 0.10$ for removal of variables. The correlations between the phase angle and traditional measures of malnutrition such as serum albumin, prealbumin, transferrin and subjective global assessment were studied using Pearson $(r)$ and Spearman's rho $(\rho)$ coefficients for normally and non-normally distributed variables respectively. For the purpose of Kaplan-Meier survival analysis, phase angle measurements were arbitrarily categorized using SPSS (SPSS Inc.) into two equal and mutually exclusive groups with a median phase angle score of $5.0^{\circ}$ as the cut-off. For Cox regression analysis, the phase angle was used as a continuous variable. 


\section{Results}

At the time of this analysis, forty-two patients had expired and sixteen were censored (reached the end of their followup without experiencing death). Tables 1 and 2 show the baseline characteristics of the patient cohort in greater detail. Table 3 shows the results of correlation analyses between phase angle and traditional indicators of malnutrition. There was a modest, but statistically significant, positive correlation between phase angle and albumin $(P<0.04)$, and between phase angle and prealbumin

Table 1. Baseline characteristics of fifty-eight pancreatic cancer patients

\begin{tabular}{|c|c|c|c|}
\hline Characteristic & Categories & $n$ & $\%$ \\
\hline \multirow[t]{2}{*}{ Gender } & Male & 35 & $60 \cdot 3$ \\
\hline & Female & 23 & $39 \cdot 7$ \\
\hline \multirow[t]{2}{*}{ Vital status } & Expired & 42 & $72 \cdot 4$ \\
\hline & Censored $^{\star}$ & 16 & $27 \cdot 6$ \\
\hline \multirow{2}{*}{$\begin{array}{l}\text { Previous treatment } \\
\text { history }\end{array}$} & Progressive disease & 32 & $55 \cdot 2$ \\
\hline & Newly diagnosed & 26 & $44 \cdot 8$ \\
\hline \multirow{4}{*}{$\begin{array}{l}\text { Subjective global } \\
\text { assessment }\end{array}$} & Well-nourished & 18 & 31 \\
\hline & Moderately malnourished & 20 & 34.5 \\
\hline & Severely malnourished & 13 & $22 \cdot 4$ \\
\hline & Unknown & 7 & $12 \cdot 1$ \\
\hline \multirow[t]{3}{*}{ Albumin } & Well-nourished ( $\geq 32 \mathrm{~g} / \mathrm{l})$ & 34 & $58 \cdot 6$ \\
\hline & Malnourished $(<32 \mathrm{~g} / \mathrm{l})$ & 19 & $32 \cdot 8$ \\
\hline & Unknown & 5 & $8 \cdot 6$ \\
\hline \multirow[t]{3}{*}{ Prealbumin } & Well-nourished ( $\geq 150 \mathrm{mg} / \mathrm{l})$ & 24 & 41.4 \\
\hline & Malnourished $(<150 \mathrm{mg} / \mathrm{l})$ & 20 & $34 \cdot 5$ \\
\hline & Unknown & 14 & $24 \cdot 1$ \\
\hline \multirow[t]{3}{*}{ Transferrin } & Well-nourished ( $\geq 2000 \mathrm{mg} / \mathrm{l})$ & 24 & $41 \cdot 4$ \\
\hline & Malnourished $(<2000 \mathrm{mg} / \mathrm{l})$ & 20 & $34 \cdot 5$ \\
\hline & Unknown & 14 & $24 \cdot 1$ \\
\hline
\end{tabular}

* Those who reached the end of their follow-up without experiencing death.

Table 2. Baseline characteristics of fifty-eight pancreatic cancer patients

(Mean values, standard deviations and ranges)

\begin{tabular}{lcccc}
\hline Characteristic & Mean & SD & Range & $\begin{array}{c}\text { Normal } \\
\text { range }\end{array}$ \\
\hline $\begin{array}{l}\text { Age at diagnosis } \\
\quad \text { (years) }\end{array}$ & $56 \cdot 2$ & $10 \cdot 7$ & $32-82$ & - \\
$\begin{array}{l}\text { Phase angle }\left(^{\circ}\right) \\
\text { Albumin }(\mathrm{g} / \mathrm{l})\end{array}$ & $5 \cdot 3$ & $1 \cdot 5$ & $2 \cdot 5-10 \cdot 1$ & - \\
$\begin{array}{l}\text { Prealbumin }(\mathrm{mg} / \mathrm{l}) \\
\text { Transferrin }(\mathrm{mg} / \mathrm{l})\end{array}$ & 165 & 66 & $70-340$ & $180-357$ \\
& 1963 & 525 & $200-3130$ & $2020-3640$ \\
\hline
\end{tabular}

Table 3. Correlations between phase angle and traditional indicators of malnutrition in advanced pancreatic cancer*

(Correlation coefficients)

\begin{tabular}{lcccr}
\hline Variables... & $\begin{array}{c}\text { Albumin } \\
(n 53)\end{array}$ & $\begin{array}{c}\text { Prealbumin } \\
(n \text { 44) }\end{array}$ & $\begin{array}{c}\text { Transferrin } \\
(n \text { 44) }\end{array}$ & $\begin{array}{c}\text { SGA } \\
(n \text { 51) }\end{array}$ \\
\hline Phase angle & 0.32 & 0.32 & 0.19 & -0.26 \\
$\begin{array}{l}\text { Statistical } \\
\text { significance: } P\end{array}$ & 0.04 & 0.04 & 0.25 & 0.10 \\
\hline
\end{tabular}

SGA, subjective global assessment.

${ }^{*}$ For details of subjects and procedures, see Tables 1 and 2 and p. 958.
$(P<0 \cdot 04)$. No statistically significant correlations were found between phase angle and transferrin, and between phase angle and subjective global assessment. Table 4 shows the univariate survival analysis of different prognostic factors. The variables are rank-ordered based on their statistical strength of association with survival. The corresponding log rank scores and $P$ values are also included. Previous treatment history had the strongest statistical strength of association with survival $(P<0.02)$. Among the nutritional indicators, phase angle was most strongly associated with survival $(P<0.03)$, followed by serum prealbumin $(P<0 \cdot 04)$. Other nutritional indicators such as serum albumin, serum transferrin and subjective global assessment were not found to be statistically significantly associated with survival time.

Fig. 1 shows the survival curves stratified by treatment history. Patients with previous treatment history had a median survival time of 4.9 (95\% CI $2.3,7.7)$ months ( $n$ 26), while newly diagnosed patients had a median survival time of $10.2(95 \%$ CI 8.8, 11.6) months $(n$ 32). The difference was statistically significant $(P=0 \cdot 02)$. Fig. 2 shows the survival curves for the two categories of phase angle. Patients with phase angle $<5.0^{\circ}$ had a median survival time of $6.3(95 \%$ CI $3.5,9.2)$ months ( $n$ 29), while those with phase angle $>5.0^{\circ}$ had a median survival time

Table 4. Univariate Kaplan-Meier survival analysis*

\begin{tabular}{lcc}
\hline Variable & $\begin{array}{c}\text { Log rank } \\
\text { score }\end{array}$ & $\begin{array}{c}\text { Statistical } \\
\text { significance: } P\end{array}$ \\
\hline Previous treatment history & 5.24 & 0.02 \\
Phase angle & 4.92 & 0.03 \\
Prealbumin & 4.34 & 0.04 \\
Subjective global assessment & 1.64 & 0.2 \\
Age & 1.22 & 0.27 \\
Transferrin & 0.55 & 0.46 \\
Albumin & 0.29 & 0.59 \\
\hline
\end{tabular}

*For details of subjects and procedures, see Tables 1 and 2 and p. 958.

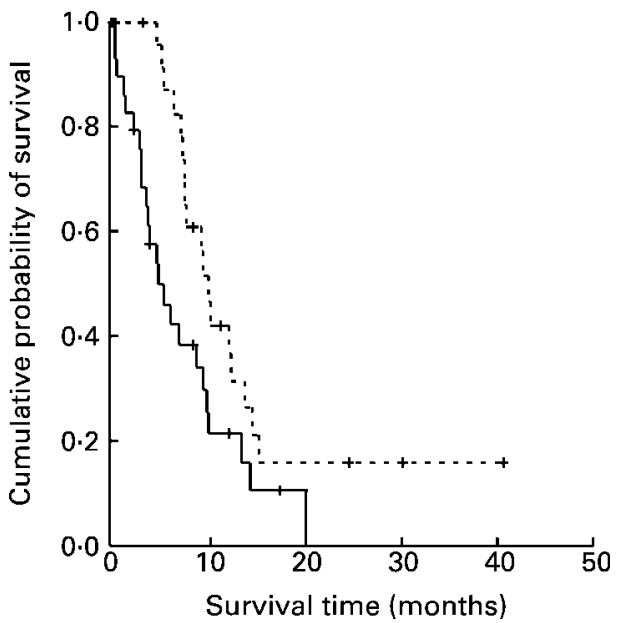

Fig. 1. Survival time stratified by previous treatment history. Each drop in a probability curve indicates one or more events in that group. - - + - -, No previous treatment; - - - previous treatment. Vertical lines indicate censored patients (those who reached the end of their follow-up without experiencing death). For details of subjects and procedures, see Tables 1 and 2 and p. 958. 


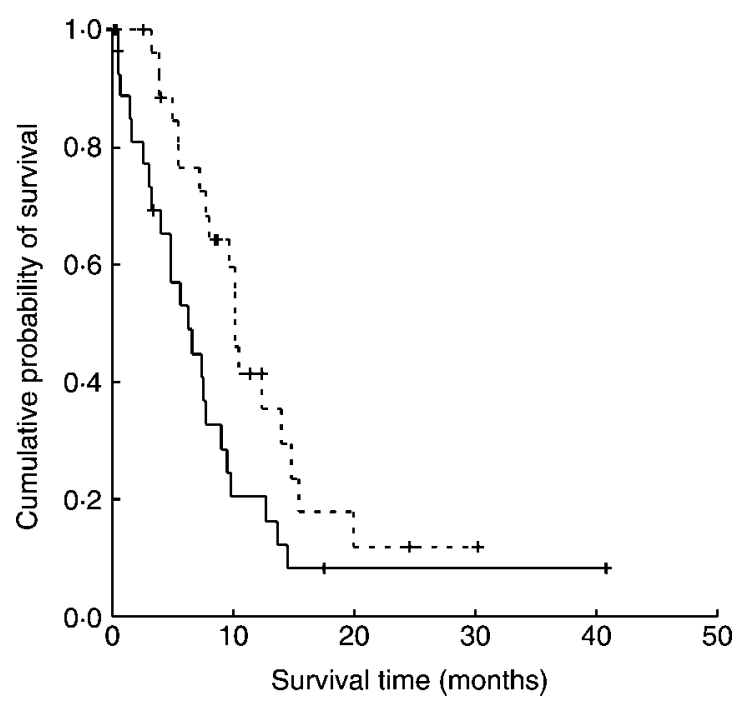

Fig. 2. Survival time stratified by phase angle categories with cutoff of $5 \cdot 0^{\circ}$. Each drop in a probability curve indicates one or more events in that group. - - + - -, High phase angle; -+- , low phase angle. Vertical lines indicate censored patients (those who reached the end of their follow-up without experiencing death). For details of subjects and procedures, see Tables 1 and 2 and p. 958.

of $10 \cdot 2(95 \%$ CI 9.6, 10.8) months (n 29). The difference was statistically significant $(P=0.02)$.

Table 5 summarizes the results of univariate and multivariate Cox regression analyses. Upon univariate Cox regression analysis with survival as the endpoint, every $1^{\circ}$ increase in phase angle was associated with a relative risk reduction of 0.75 (95\% CI $0.58,0.96)$. Multivariate Cox modelling, after adjusting for prealbumin, demonstrated a relative risk reduction of 0.69 (95\% CI 0.49 , 0.97 ) associated with every $1^{\circ}$ increase in phase angle. Interestingly, phase angle continued to be statistically significant $(P=0.02)$ after jointly controlling for serum albumin, prealbumin and previous treatment history. Cox regression modelling using the backward elimination

Table 5. Univariate and multivariate Cox proportional hazards model $^{*}$

\begin{tabular}{llll}
\hline $\begin{array}{l}\text { Independent } \\
\text { variable(s) }\end{array}$ & $\mathrm{RR}$ & $95 \% \mathrm{Cl}$ & $\begin{array}{c}\text { Statistical } \\
\text { significance: } P\end{array}$ \\
\hline $\begin{array}{l}\text { Univariate model } \\
\text { Phase angle }\end{array}$ & 0.75 & $0.58,0.96$ & 0.02 \\
$\begin{array}{l}\text { Multivariate models } \\
\text { 1. Phase angle }\end{array}$ & 0.69 & $0.49,0.97$ & 0.03 \\
$\quad$ Prealbumin & 1.10 & $0.99,1.12$ & 0.09 \\
2. Phase angle & 0.78 & $0.60,1.00$ & 0.07 \\
$\quad$ Albumin & 0.59 & $0.30,1.15$ & 0.12 \\
3. Phase angle & 0.80 & $0.62,1.02$ & 0.07 \\
$\quad$ Treatment history & 1.80 & $0.93,3.40$ & 0.08 \\
4. Phase angle & 0.64 & $0.45,0.92$ & 0.02 \\
$\quad$ Prealbumin & 1.10 & $1.03,1.20$ & 0.01 \\
$\quad$ Albumin & 0.37 & $0.13,1.10$ & 0.07 \\
$\quad$ Treatment history & 1.62 & $0.72,3.60$ & 0.24 \\
5. Phase angle & 0.64 & $0.45,0.91$ & 0.01 \\
$\quad$ Prealbumin & 1.10 & $1.02,1.20$ & 0.01 \\
$\quad$ Albumin & 0.37 & $0.13,1.04$ & 0.06 \\
\hline
\end{tabular}

$\mathrm{RR}$, relative risk.

${ }^{*}$ For details of subjects and procedures, see Tables 1 and 2 and p. 958. likelihood ratio method retained phase angle, prealbumin and albumin in the final model as predictors of patient survival.

\section{Discussion}

The identification of prognostic factors in advanced pancreatic cancer is of considerable importance for clinical management of the disease. While nutritional status has been hypothesized to have an association with survival, the published literature documenting its prognostic significance in advanced pancreatic cancer remains sparse. The current study was undertaken to investigate whether the BIA-derived phase angle, a potential indicator of nutritional status, could predict survival in advanced pancreatic cancer.

The present study demonstrated that phase angle is a more powerful predictor of survival than the traditional nutritional indices such as albumin, prealbumin and transferrin in patients with advanced pancreatic cancer. A similar study conducted in patients with advanced lung cancer stratified the patient cohort by mean phase angle $4.5^{\circ}$. Interestingly, patients with phase angle $\leq 4.5^{\circ}$ had a significantly shorter survival than those with phase angle $>4.5^{\circ}$ (Toso et al. 2000). Another study conducted in HIVinfected patients stratified patients into four quartiles, with $5.3^{\circ}, 5.9^{\circ}$ and $6.5^{\circ}$ as the cut-off points. The study found phase angle to be an independent prognostic marker of clinical progression and survival (Schwenk et al. 2000). In another prospective study of patients with liver cirrhosis, phase angle $\leq 5.4^{\circ}$ was associated with shorter survival compared with phase angle $>5.4^{\circ}$ (Selberg $\&$ Selberg, 2002). Similarly, a prospective study conducted in forty-eight peritoneal dialysis patients found that phase angle $<6^{\circ}$ was an adverse predictor of survival time (Mushnick et al. 2003).

The present study raises several important but complex questions. Can phase angle be considered to be a surrogate marker of an underlying nutritional or metabolic disorder? Can phase angle be used as a nutritional assessment tool in advanced cancer? Can phase angle be altered by any direct or indirect medical or nutritional intervention with a subsequent improvement in prognosis? Some earlier studies have tried to address these questions in limited capacity. For instance, Schwenk et al. (2000) hypothesized that phase angle could be interpreted as a global marker of malnutrition in HIV-infected patients. In another study conducted on HIV-infected patients, it was argued that phase angle reflects the integrity of vital cell membranes (Ott et al. 1995). Selberg \& Selberg (2002), who investigated whether phase angle could be adequate for nutritional assessment in patients with liver cirrhosis, speculated that phase angle could be a marker of clinically relevant malnutrition characterized by both increased extracellular mass and decreased body cellular mass. In advanced lung cancer, phase angle was speculated to be an indicator of altered tissue electrical properties (Toso et al. 2000).

As a step to further understand the clinical applications of phase angle assessment, we propose that future studies should try to address the following issues. Can phase angle be used as a prognostic indicator in patients with 
other cancer types, such as breast and colo-rectal? Does phase angle continue to be a predictor of survival after patients undergo definitive treatment for their advanced cancer? Can nutritional intervention have any effect on phase angle that could possibly improve patient survival? In addition, the use of phase angle as a nutritional assessment tool needs to be validated in a variety of cancer populations, because the underlying assumptions relevant to one type of cancer might not hold true for another. The present study provides a good starting point for future research in these directions.

Limitations of the present study relate to the BIA technique and retrospective study design. The present study, because of its retrospective nature, relies on data not primarily meant for research. One potential limitation of the BIA approach is the reliance on regression models, derived in restricted samples of human subjects, which restricts the usefulness of the derived model in other patients who differ from the original sample in which the model was developed (Elia, 1993; Lukaski, 1999). However, in our present study, we evaluated phase angle, which does not depend on regression equations to be calculated, thereby eliminating a large source of random error (Barbosa-Silva et al. 2003). It has also been suggested that the variability of direct bioimpedance measures (resistance, capacitance and phase angle) depends on age, gender and body mass characteristics of the study population; this may limit the validity of extrapolation of the model (Elia, 1993; Dittmar, 2003). In a review paper, Foster \& Lukaski (1996) argued that although the correlation between whole-body impedance measurements and body composition is experimentally well established, the reason for the success of the impedance technique is much less clear.

Some other reported limitations of using BIA for assessment of body composition are hydration status and/or major disturbances of water distribution, body position during procedure, ambient air and skin temperatures, recent physical activity, conductance of the examining table and food consumption (Anon, 1996). Since the original intent of the BIA in the present study was to gather estimates of body composition as part of a baseline nutritional assessment in a clinical setting, not all of these factors could realistically be controlled. Patients were free of visible oedema or ascites, so there was control for obvious over-hydration. Body position was controlled because all patients were in the supine position in a bed or on an examination table. Air temperature was within a controlled range in our hospital setting. Physical activity was limited in these patients due to the advanced nature of their disease. Finally, food intake was not controlled in this clinical setting, which may have contributed to a small amount of variability.

The present study did not evaluate the prognostic significance of alternative nutritional indicators such as C-reactive protein and mid-arm muscle circumference because of the lack of data on these variables. These indicators have been shown to be more practical, better understood biologically, more strongly prognostic and better indicators of nutritional status than the phase angle. The cut-off point for the phase angle in the present study was generated so as to arbitrarily divide the patient population into two equal and mutually exclusive groups. Although our cut-off point was in agreement with those reported by other researchers in the field (Chertow et al. 1997; Schwenk et al. 2000; Toso et al. 2000; Selberg \& Selberg, 2002), there is a clear need to define threshold values for the phase angle as a nutritional assessment tool based on large prospective studies in advanced cancer. For example, a study conducted in a large sample of haemodialysis patients found a mean phase angle of $4.82^{\circ}$ compared with a mean value of $5.3^{\circ}$ in our present study (Chertow et al. 1997). We also think that restricting the analysis to newly diagnosed patients (patients with no previous treatment history) would have been more accurate, since it would have allowed for evaluation of true overall survival time, i.e. time from the date of diagnosis to the date of death. However, doing so would have caused a significant reduction in the sample size. In our present study, the survival time was calculated from the day of first patient visit at our hospital because the BIA measurements were not available at the time of diagnosis for previously treated patients. This limitation emphasizes the need for conducting prospective studies, which have nutritional information available since the date of diagnosis. No assessment of inter-rater reliability of the users of BIA was made in the present study. This bias, however, was minimized by restricting the use of BIA to well-trained dietitians with an expertise in the use of this clinical technique. All dietitians received a minimum of 2 weeks training in the BIA technique during their orientation to the department.

In summary, our present study has demonstrated the prognostic significance of the phase angle in advanced pancreatic cancer. To the best of our knowledge this is the first study to evaluate phase angle for its prognostic importance in advanced pancreatic cancer.

\section{Acknowledgements}

This study was supported by Cancer Treatment Centers of America ${ }^{\circledR}$. We are grateful to Gwendolynn Lambert for assistance with data collection for this project.

\section{References}

Adami GF, Marinari G, Gandolfo P, Cocchi F, Friedman D \& Scopinaro N (1993) The use of bioelectrical impedance analysis for monitoring body composition changes during nutritional support. Surg Today 23, 867-870.

Anon (1996) Bioelectrical impedance analysis in body composition measurement: National Institutes of Health Technology Assessment Conference Statement. Am J Clin Nutr 64, $524 \mathrm{~S}-532 \mathrm{~S}$.

Azcue M, Fried M \& Pencharz PB (1993) Use of bioelectrical impedance analysis to measure total body water in patients with cystic fibrosis. J Pediatr Gastroenterol Nutr 16, 440-445.

Barbosa-Silva MC, Barros AJ, Post CL, Waitzberg DL \& Heymsfield SB (2003) Can bioelectrical impedance analysis identify malnutrition in preoperative nutrition assessment? Nutrition 19, 422-426.

Bauer J, Capra S \& Ferguson M (2002) Use of the scored PatientGenerated Subjective Global Assessment (PG-SGA) as 
a nutrition assessment tool in patients with cancer. Eur J Clin Nutr 56, 779-785.

Baumgartner RN, Chumlea WC \& Roche AF (1988) Bioelectric impedance phase angle and body composition. Am J Clin Nutr 48, 16-23.

Bohmig M \& Rosewicz S (2004) Pancreatic carcinoma. Z Gastroenterol 42, 261-268.

Carney DE \& Meguid MM (2002) Current concepts in nutritional assessment. Arch Surg 137, 42-45.

Chertow GM, Lazarus JM, Lew NL, Ma L \& Lowrie EG (1997) Bioimpedance norms for the hemodialysis population. Kidney Int 52, 1617-1621.

Chertow GM, Lowrie EG, Wilmore DW, Gonzalez J, Lew NL, Ling J, Leboff MS, Gottlieb MN, Huang W \& Zebrowski B (1995) Nutritional assessment with bioelectrical impedance analysis in maintenance hemodialysis patients. J Am Soc Nephrol 6, 75-81.

Desport JC, Preux PM, Bouteloup-Demange C, Clavelou P, Beaufrere B, Bonnet C \& Couratier PP (2003) Validation of bioelectrical impedance analysis in patients with amyotrophic lateral sclerosis. Am J Clin Nutr 77, 1179-1185.

Detsky AS, Baker JP, Mendelson RA, Wolman SL, Wesson DE \& Jeejeebhoy KN (1984) Evaluating the accuracy of nutritional assessment techniques applied to hospitalized patients: methodology and comparisons. J Parenter Enteral Nutr 8, 153-159.

Dittmar M (2003) Reliability and variability of bioimpedance measures in normal adults: effects of age, gender, and body mass. Am J Phys Anthropol 122, 361-370.

Elia M (1993) The bioimpedance 'craze'. Eur J Clin Nutr 47, $825-827$.

Faisy C, Rabbat A, Kouchakji B \& Laaban JP (2000) Bioelectrical impedance analysis in estimating nutritional status and outcome of patients with chronic obstructive pulmonary disease and acute respiratory failure. Intensive Care Med 26, 518-525.

Fein PA, Gundumalla G, Jorden A, Matza B, Chattopadhyay J \& Avram MM (2002) Usefulness of bioelectrical impedance analysis in monitoring nutrition status and survival of peritoneal dialysis patients. Adv Perit Dial 18, 195-199.

Foster KR \& Lukaski HC (1996) Whole-body impedance - what does it measure? Am J Clin Nutr 64, 388S-396S.

Fredrix EW, Saris WH, Soeters PB, Wouters EF, Kester AD, von Meyenfeldt MF \& Westerterp KR (1990) Estimation of body composition by bioelectrical impedance in cancer patients. Eur J Clin Nutr 44, 749-752.

Higashiguchi T, Kita T, Noguchi T, Kawarada Y \& Mizumoto R (1988) Importance of nutritional management for the treatment of carcinoma of the pancreas. Gan To Kagaku Ryoho 15, 847-853.

Lukaski HC (1999) Requirements for clinical use of bioelectrical impedance analysis (BIA). Ann NY Acad Sci 873, 72-76.

Lukaski HC, Johnson PE, Bolonchuk WW \& Lykken GI (1985) Assessment of fat-free mass using bioelectrical impedance measurements of the human body. Am J Clin Nutr 41, $810-817$.

Maggiore Q, Nigrelli S, Ciccarelli C, Grimaldi C, Rossi GA \& Michelassi C (1996) Nutritional and prognostic correlates of bioimpedance indexes in hemodialysis patients. Kidney Int 50, 2103-2108.

(50, $2103-2108$.
Marken Lichtenbelt WD, Westerterp KR, Wouters L \& Luijendijk SC (1994) Validation of bioelectrical-impedance measurements as a method to estimate body-water compartments. Am J Clin Nutr 60, 159-166.

Mushnick R, Fein PA, Mittman N, Goel N, Chattopadhyay J \& Avram MM (2003) Relationship of bioelectrical impedance parameters to nutrition and survival in peritoneal dialysis patients. Kidney Int 83, Suppl., S53-S56.

Nagano M, Suita S \& Yamanouchi T (2000) The validity of bioelectrical impedance phase angle for nutritional assessment in children. J Pediatr Surg 35, 1035-1039.

Ott M, Fischer H, Polat H, Helm EB, Frenz M, Caspary WF \& Lembcke B (1995) Bioelectrical impedance analysis as a predictor of survival in patients with human immunodeficiency virus infection. J Acquir Immune Defic Syndr Hum Retrovirol 9, 20-25.

Ottery F (1996) Supportive nutritional management of the patient with pancreatic cancer. Oncology (Huntingt) 10, 26-32.

Pencharz PB \& Azcue M (1996) Use of bioelectrical impedance analysis measurements in the clinical management of malnutrition. Am J Clin Nutr 64, 485S-488S.

Pupim LB, Kent P \& Ikizler TA (1999) Bioelectrical impedance analysis in dialysis patients. Miner Electrolyte Metab 25, 400-406.

Rocha Lima CM \& Centeno B (2002) Update on pancreatic cancer. Curr Opin Oncol 14, 424-430.

Sarhill N, Mahmoud FA, Christie R \& Tahir A (2003) Assessment of nutritional status and fluid deficits in advanced cancer. Am J Hosp Palliat Care 20, 465-473.

Schwenk A, Beisenherz A, Romer K, Kremer G, Salzberger B \& Elia M (2000) Phase angle from bioelectrical impedance analysis remains an independent predictive marker in HIV-infected patients in the era of highly active antiretroviral treatment. Am J Clin Nutr 72, 496-501.

Schwenk A, Ward LC, Elia M \& Scott GM (1998) Bioelectrical impedance analysis predicts outcome in patients with suspected bacteremia. Infection 26, 277-282.

Selberg O \& Selberg D (2002) Norms and correlates of bioimpedance phase angle in healthy human subjects, hospitalized patients, and patients with liver cirrhosis. Eur J Appl Physiol 86, 509-516.

Simons JP, Schols AM, Westerterp KR, ten Velde GP \& Wouters EF (1995) The use of bioelectrical impedance analysis to predict total body water in patients with cancer cachexia. Am J Clin Nutr 61, 741-745.

Talluri T, Lietdke RJ, Evangelisti A, Talluri J \& Maggia G (1999) Fat-free mass qualitative assessment with bioelectric impedance analysis (BIA). Ann NY Acad Sci 873, 94-98.

Toso S, Piccoli A, Gusella M, Menon D, Bononi A, Crepaldi G \& Ferrazzi E (2000) Altered tissue electric properties in lung cancer patients as detected by bioelectric impedance vector analysis. Nutrition 16, 120-124.

Waitzberg DL \& Correia MI (2003) Nutritional assessment in the hospitalized patient. Curr Opin Clin Nutr Metab Care 6, 531-538.

Zarowitz BJ \& Pilla AM (1989) Bioelectrical impedance in clinical practice. DICP 23, 548-555. 